\title{
Discusiones Metodológicas en el Estudio del Desarrollo de la Intencionalidad Compartida en Niños: Una Revisión Sistemática
}

\section{Methodological Discussions in the Study of the Development of Shared Intentionality in Children: A Systematic Review}

\author{
Juan Felipe Velásquez, Erika Johnnela Gómez, Ximena Restrepo, \\ Edward Chávez, Juan David Piñeres y Johny Villada \\ Universidad de Antioquia
}

\begin{abstract}
La investigación sobre las habilidades cognitivas de los niños que sustentan la intencionalidad compartida viene desarrollándose con mucha fuerza en los últimos años. El presente artículo contiene una discusión sobre las metodologías usadas en el campo, para lo cual se revisaron investigaciones publicadas entre 2005 y 2017 cuyo tema principal es la intencionalidad compartida. Como resultado, se propone una distinción general entre 2 posturas: aquellas metodologías que son activas o que requieren respuestas conductuales que incluyen movimiento por parte del infante y aquellas que son pasivas o que se basan en las reacciones visuales o emocionales del niño. Se presenta un análisis crítico del uso de dichas metodologías, de sus alcances y limitaciones. Finalmente, se propone que las tensiones y discusiones a nivel metodológico pueden tener de fondo diferencias teóricas respecto al abordaje del fenómeno.
\end{abstract}

Palabras clave: intencionalidad compartida, desarrollo, metodologías pasivas, metodologías activas

\begin{abstract}
Research on children's cognitive abilities yielding evidence in support of shared intentionality has greatly increased in recent years. The present article discusses the methodologies used in the field by reviewing research published between 2005 and 2017 whose main topic was shared intentionality. As a result, a distinction between 2 approaches is proposed: those methodologies that are active or require behavioral responses that include infant movement and those that are passive or are based on children's visual or emotional responses. A critical analysis of the use of these methodologies, their implications, and their limitations is presented. Finally, it is proposed that tensions and discussions at the methodological level may be due to theoretical differences regarding the study of the phenomenon.
\end{abstract}

Keywords: shared intentionality, development, passive methodologies, active methodologies

En 2005, Tomasello, Carpenter, Call, Behne y Moll plantearon la intencionalidad compartida como la adaptación fundamental que sustenta la evolución cultural propia de la especie humana. Se trata de la capacidad para participar con otros de actividades colaborativas en las que se comparten metas e intenciones, las cuales se alcanzan por medio de la estructuración de planes de acción que incluyen roles diferenciados entre los participantes. Según Tomasello et al. (2005), las metas se definen como la representación mental de un estado del mundo que es deseado por el sujeto y que guía la conducta; las intenciones se entienden como el plan de acción elegido para conseguir dicha meta. Por ejemplo, si el sujeto se encuentra ante una ventana abierta y se dirige a cerrarla, entendemos como meta la representación mental de esa ventana cerrada y como intención, la conducta específica que elige y realiza el sujeto para cerrar la ventana (e.g., ir a la ventana y cerrarla, usar un palo para cerrar la ventana, pedirle a alguien que cierre la ventana). Nótese que la intención debe ir siempre acompañada de una meta, aunque una meta no necesariamente incluye un plan de acción.

La intencionalidad compartida requiere que dos o más sujetos tengan (a) metas compartidas y (b) capacidad para comprender las intenciones de los demás. Una meta compartida es una representación de un estado del mundo deseado por dos o más sujetos, donde la meta de cada participante incluye contenido de las metas de los otros (Tomasello et al., 2005). Por ejemplo, un cuarteto de cuerdas tiene como meta compartida interpretar el Canon de Pachelbel; para ello, es necesario que la meta del chelista incluya contenido de la meta del violista y de cada uno de los violinistas y así sucesivamente con cada uno de los

Juan Felipe Velásquez Jaramillo, Erika Johnnela Gómez, Ximena Restrepo, Edward Chávez, Juan David Piñeres Sus y Johny Villada Zapata, Departamento de Psicología, Universidad de Antioquia, Medellín, Colombia.

La correspondencia relativa a este artículo debe dirigirse a Johny Villada Zapara, Departamento de Psicología, Universidad de Antioquia, Calle 67 \#53, 108 Medellín, Antoquia, Colombia. Email: johny.villada@udea.edu.co 
intérpretes. A su vez, la intencionalidad compartida requiere que cada uno de los participantes presente habilidades cognitivas relacionadas con la comprensión de intenciones, de forma que cada participante conozca y prevea los planes de los demás, coordinando la acción conjunta. Así, en el cuarteto de cuerdas, el chelista conoce el rol del violinista y realiza su acción teniendo en cuenta la intención del violinista; si cada músico realiza lo propio, el resultado será una interpretación coordinada del Canon de Pachelbel.

En el marco de la intencionalidad compartida, autores como Carpenter, Call y Tomasello (2005) señalan que, en las investigaciones sobre el desarrollo de la comprensión de acciones intencionales, se han presentado resultados distintos respecto de las habilidades estudiadas, debido, principalmente, a una dificultad respecto del establecimiento de una conducta observable que permita a los investigadores inferir cuándo y cuándo no un niño comprende las intenciones subyacentes de los agentes involucrados en una situación. Estos autores plantean un problema metodológico evidente: el surgimiento de dicha capacidad depende del método de medición utilizado en cada investigación.

Dado lo anterior, debido a que la comprensión de intenciones pertenece a un marco de referencia más amplio, se propone una revisión de las investigaciones sobre la intencionalidad compartida posteriores al 2005, con el fin de analizar los diversos métodos utilizados y comprender qué ha sucedido con el problema denunciado por Carpenter et al. (2005).

\section{Método}

En este artículo se realiza una discusión metodológica sobre el estudio del desarrollo de la comprensión de intenciones, a partir de una investigación tipo revisión sistemática en la que se encontraron 86 documentos y se descartaron 28, dando como resultado 58 artículos publicados entre 2005 y 2017 relacionados con el tema, con los cuales se trabajó. Se descartaron los artículos que no cumplían cabalmente con los criterios de inclusión. Las bases de datos consultadas fueron Science Direct, Ebsco, Dialnet, Scielo, Jstor y Scopus. Para hacer esta revisión sistemática se realizaron búsquedas hasta octubre de 2017, luego fue llevado a cabo un proceso de selección de los artículos y su respectivo fichaje y análisis. Como palabras clave en la búsqueda documental se utilizaron las siguientes: shared intentionality, animated action, goal-directed action, joint attention y rational imitation. También se utilizaron palabras en español, como intencionalidad compartida, acciones animadas, acciones dirigidas a metas, atención conjunta e imitación racional; sin embargo, no se encontraron artículos relacionados con el tema en esta lengua. El análisis consistió en la lectura crítica de cada artículo, la elaboración de una ficha resumen que incluía aspectos relevantes del artículo en términos metodológicos y teóricos y la discusión semanal de los avances encontrados.

Como criterios de inclusión se tuvieron en cuenta artículos propios de la psicología del desarrollo humano, es decir, aquellos que indagan por la aparición y el cambio en las variables estudiadas (aunque algunas referencias incluídas realizan estudios comparados) y que estuvieran publicados en revistas indexadas entre los años 2005 y 2017. Los criterios de exclusión fueron reseñas de libros, boletines, perspectivas o comentarios que no se derivaran de investigaciones y publicaciones en revistas indexadas. A continuación, se presentan los principales elementos a discutir sobre la metodología de las investigaciones consultadas.

\section{Resultados}

\section{Metodologías de Investigación Según su Demanda Motora: Activas y Pasivas}

A partir de la presente revisión, se propone que las metodologías usadas para estudiar las habilidades que componen la intencionalidad compartida pueden ser, a grandes rasgos, agrupadas en activas y pasivas. En la Tabla 1 y 2 se presenta un resumen de los artículos revisados y su clasificación según la metodología propuesta, así como los métodos utilizados en cada uno de ellos. Grosso modo, las investigaciones cuentan con un promedio de 41,2 participantes con una edad promedio de 15,3 meses; el rango de edad va desde los 2 meses hasta los 60 meses (5 años), encontrándose una diferencia de edades según la metodología utilizada. En las metodologías pasivas, los estudios se centran en edades desde los 2 a los 24 meses; por el contrario, las metodologías activas van desde los 12 a los 60 meses, siendo predominantes los estudios con niños de 36 a 60 meses. Otro aspecto a resaltar es que las investigaciones incluidas corresponden a estudios con infantes humanos; solo tres investigaciones $(5,36 \%)$ reportan estudios con primates no humanos. 
Tabla 1

Resumen de Metodologías Empleadas en los Estudios

\begin{tabular}{lc}
\hline \multicolumn{1}{c}{ Metodología } & Porcentaje \\
\hline Activa & 38,5 \\
Activa/pasiva & 1,9 \\
Pasiva & 59,6 \\
Total & 100,0 \\
\hline
\end{tabular}

Tabla 2

Resumen de Métodos Específicos Usados en los Estudios

\begin{tabular}{lc}
\hline \multicolumn{1}{c}{ Método } & Porcentaje \\
\hline Habituación visual & 44,2 \\
Habituación visual/imitación & 1,9 \\
Irrelevant actions & 23,1 \\
Still-face effect & 11,5 \\
Teórico & 3,8 \\
Unsuccessful actions & 15,4 \\
Total & 100,0 \\
\hline
\end{tabular}

Por un lado, en las metodologías activas el movimiento y la interacción del infante con la situación funcionan como indicadores de la habilidad estudiada (e.g., imitación, conductas de ayuda, gestos comunicativos). Por otro lado, las metodologías pasivas asumen como indicadores las reacciones visuales y/o emocionales del infante frente a ciertas situaciones (e.g., habituación visual, preferencia de objeto, seguimiento de la mirada, violación de expectativa). Así, la principal diferencia recae en la demanda motora que se le exige al infante para responder a las situaciones experimentales, siendo menor en las metodologías pasivas.

El tipo de metodología pasiva ha permitido medir una serie de habilidades que antes no se concebían en momentos tempranos del desarrollo y que resultaban complicadas de evaluar de otras formas, por las altas demandas motoras o lingüísticas al infante (Puche-Navarro, 2000). Por esa razón, las metodologías pasivas han sido predilectas en el estudio del surgimiento de habilidades cognitivas en edades tempranas del desarrollo, pues el infante no requiere de un desarrollo amplio de la motricidad o el lenguaje —entre otros procesos - para responder a la situación que está evaluando la habilidad estudiada. Müller y Giesbrecht (2008) muestran esta misma tendencia en el estudio de las habilidades relacionadas con el razonamiento y la representación; asimismo, en relación con las habilidades de la intencionalidad compartida, la revisión realizada por Marsh y Legerstee (2017) muestra cómo las metodologías pasivas han sido útiles para medir el surgimiento del seguimiento de mirada, pero han presentado dificultades cuando se pretende evaluar habilidades más complejas. Las dificultades recaen en que las respuestas que usan dichas metodologías para inferir una habilidad no constituyen un indicador directo de la misma. Dicho de otro modo, para afirmar la existencia de capacidades o habilidades cognitivas las metodologías pasivas exigen respuestas menos demandantes al niño, pero requieren un proceso de inferencia complejo que parte de una interpretación de la respuesta del infante, más no de la conducta observable. Así, por ejemplo, un sesgo atencional por parte del infante puede ser interpretado como una violación de expectativa, como si el niño tuviese un entendimiento de que algo no es usual. Este entendimiento indicaría — para algunos autores- la comprensión de intenciones.

Las metodologías activas, al no requerir de un proceso de inferencia tan complejo, se han tomado como una forma de medición más precisa de la habilidad que se esté estudiando. De esta manera, si el niño responde a la tarea experimental, su comportamiento se interpreta como un indicador directo de la habilidad. Sin embargo, estas metodologías incluyen una exigencia alta del niño, que resulta ser, a su vez, una limitación 
para el estudio de las habilidades en momentos más tempranos del desarrollo. Es posible que, si se le exigen al infante procesos más complejos que no estén relacionados directamente con lo que se está estudiando, la falla en la resolución de la tarea experimental no se deba, necesariamente, a la ausencia de la habilidad, sino a la complejidad de la tarea.

En lo que sigue, se realiza un análisis de las metodologías recurrentes en el estudio del desarrollo de la comprensión de intenciones. Para ello, se ilustran algunas discusiones respecto del alcance y limitaciones de metodologías pasivas — habituación visual y still-face effect- y metodologías activas —unsuccessful actions e irrelevant actions-. Además, se presentan consideraciones sobre la interpretación de los datos.

\section{Metodologías Pasivas.}

Habituación visual. Algunas metodologías que se basan en la conducta visual parten de una comparación del tiempo de mirada entre dos situaciones, es decir, se valen de un sesgo atencional (mirar más una situación que otra) para inferir la discriminación entre situaciones (Delafield-Butt \& Gangopadhyay, 2013; Hamlin, Newman \& Wynn 2009; Henderson \& Woodward, 2011; Hernik \& Southgate, 2012; Marsh \& Legerstee, 2017; Thoermer, Woodward, Sodian, Perst \& Kristen, 2013). En el entendimiento de acciones dirigidas a metas, la metodología de habituación visual tiene variaciones entre estudios, aunque, grosso modo, en un primer momento consiste en presentar una situación en la que un actor interactúa con uno de dos objetos disponibles en la escena. En un segundo momento, luego de que el niño se ha habituado a la acción, se altera la escena y se mide el tiempo de mirada del niño: una mayor duración indica que se ha violado la expectativa del infante respecto de la acción realizada. Así, si los niños miran más cuando se altera la escena - y la acción realizada por el actor-, se infiere que hay una comprensión de acciones dirigidas a metas. Según los autores, como la meta es, aparentemente, la única diferencia presente entre ambas situaciones, el sesgo atencional da cuenta de la presencia de la habilidad de comprensión de acciones dirigidas a metas, incluso en edades tempranas (Gredebäck, Fikke \& Melinder, 2010; Kim \& Song, 2015; Marsh, Stavropoulos, Nienhuis \& Legerstee, 2010; Scott \& Baillargeon, 2013).

Aunque las conclusiones son cuestionables, lo cierto es que los niños distinguen entre las situaciones. Sin embargo, es posible que dicho sesgo atencional se deba a otras causas más simples que al entendimiento de metas, por ejemplo, a la habituación a patrones comportamentales conocidos. Tomasello et al. (2005) exponen que, alrededor de los seis meses, los niños ya han formado suficientes expectativas sobre las acciones humanas como para poder predecir qué hará el otro en una situación familiar. Además, se ha demostrado cómo los niños esperan que, durante una misma situación, los adultos vuelvan a ejecutar la acción que han estado realizando previamente sobre un objeto (Adam et al., 2016; Feiman, Carey \& Cushman, 2015; JowkarBaniani, Paolozza, Greene, Cheng \& Schmuckler, 2017; Olofson \& Baldwin, 2011; Ransburg, Reiser, Munzert, Jovanovic \& Schwarzer, 2017; Woodward, 1998). Esta expectativa no necesariamente debe interpretarse como un entendimiento de que las acciones del adulto están dirigidas a metas, sino como un entendimiento de que esas acciones no son consistentes con el patrón comportamental que el adulto ha presentado hasta el momento.

Para solventar el problema de la distinción entre situaciones, y partiendo también de una metodología de habituación visual, varios autores (Biro \& Leslie, 2007; Luo, 2011; Luo \& Baillargeon, 2005) plantean que niños muy pequeños (de tres a seis meses) atribuyen metas a cualquier agente — animado o inanimado-, siempre y cuando sus acciones - conocidas o novedosas- cumplan tres características: (a) tener autopropulsión (self-propelledness); (b) presentar variaciones equifinales (equifinal variations), es decir, alcanzar la misma meta realizando la misma acción, pero con modificaciones en su trayectoria, alcance o contacto y (c) que se presenten efectos en los objetos después de realizar las acciones sobre ellos (action-effect). Los resultados indican que los niños reconocen la diferencia entre acciones ambiguas y acciones dirigidas a metas, tanto en patrones comportamentales conocidos como en comportamientos novedosos. Sin embargo, de nuevo, los datos se pueden explicar recurriendo a mecanismos más simples: es posible que los niños anticipen las acciones de los agentes, porque han establecido una asociación entre el agente y el objeto, sin necesidad de entender la existencia de una meta que motive al agente. De esta forma, el sesgo atencional en el tiempo de mirada puede deberse a una violación de expectativa, porque el agente y el objeto asociados no han interactuado como solían hacerlo y no a una violación de expectativa por el no cumplimiento de la meta del agente.

Paulus (2011) demuestra experimentalmente que los resultados de los estudios que usan habituación visual se explican por el desarrollo de mecanismos de aprendizaje asociativo y no por el surgimiento de un entendimiento de acciones dirigidas a metas. El autor afirma que el tiempo de mirada no es un indicador 
fiable del entendimiento de metas y, en su lugar, propone la medición con anticipatory gaze-shift; dicha medición consiste en observar si el niño mira el lugar u objeto al que debería dirigirse la acción de un agente (usualmente a través de eye-tracking), lo cual permite inferir que el niño entiende metas porque anticipa la acción del agente antes de que ocurra. Los resultados de su estudio indican que, en primer lugar, los niños establecen asociaciones entre agente y objeto, independientemente de la acción que uno realice sobre el otro. Esta asociación conlleva el aumento del tiempo de mirada después de presentar el resultado de la acción, pero no conduce a que se presenten miradas anticipatorias hacia el resultado esperado (anticipatory gaze-shifts), por lo que en estos infantes se puede establecer una atribución de agencia, pero no de acciones dirigidas a metas. En segundo lugar, se demuestra que la mera presencia simultánea del agente y del objeto genera un reflejo por asociación que aumenta el tiempo de mirada; por tanto, no se requiere de ninguna interacción entre agente y objeto para generar una asociación entre ellos, mucho menos la existencia de una meta.

Otras metodologías basadas en la conducta visual hacen uso del paradigma de eye-tracking para estudiar el desarrollo de habilidades comunicativas no verbales asociadas a la intencionalidad compartida, tanto durante interacciones diádicas, de niño-adulto (Devouche, Dominguez, Bobin-Bègue, Gratier \& Apter, 2012; Perra \& Gattis, 2012), como triádicas, de niño-objeto-adulto (Brandone, 2015; Gredebäck et al., 2010; Tremblay \& Rovira, 2007). En las interacciones triádicas, esto es, cuando sigue la dirección de mirada del adulto hacia un objeto o cuando alterna la mirada entre el objeto y el adulto, los autores suelen inferir que el niño participa en situaciones de atención conjunta, incluso en edades tempranas (entre los tres y seis meses). De ese modo, las investigaciones únicamente dan cuenta de una de las habilidades que compone la atención conjunta: responder a la atención (Gredebäck et al., 2010); y no incluyen la habilidad de iniciar la atención conjunta, habilidad que implica que el niño entienda al adulto como un agente intencional cuya atención puede ser dirigida hacia un objeto o evento específico (ver al respecto, Beuker, Rommelse, Donders \& Buitelaar, 2013). Además, el cambio de la dirección de la mirada del niño puede explicarse como una reacción conductual dada por un objeto que ingresa al campo visual, lo que no implica que entienda que hay una referencia del adulto hacia este objeto como algo compartido por ellos. Por medio de metodologías alternativas (e.g., pointing, imitation, hand-eye coordination), otras investigaciones concluyen que los niños participan en situaciones de atención conjunta (i.e., siguen y dirigen las invitaciones de compartir la atención) en edades más tardías (Mundy et al., 2007; Yu \& Smith, 2013, 2017).

El desarrollo de la comprensión de acciones intencionales se consolida sobre habilidades que tienen un gran componente cognitivo e implicaciones sociales. Si bien el uso de metodologías como la habituación visual y el paradigma de eye-tracking permite estudiar habilidades pre-verbales y que no incluyan demandas motoras mayores, en muchas ocasiones la conducta visual del niño puede ser explicada por el desarrollo de habilidades cognitivas que son menos complejas que las propuestas por los autores.

Still face effect. Otra metodología que se ha usado con niños muy pequeños para inferir entendimiento de intenciones en la medición de diferentes reacciones emocionales de los infantes frente a las acciones del adulto es el still-face effect (Marsh et al., 2010; Mastergeorge, Paschall, Loeb \& Dixon, 2014). Consiste en que un adulto detiene súbitamente los gestos y articulaciones que estaba haciendo frente el niño, asumiendo una postura y un rostro neutral. Ante esta situación, la reacción usual de los infantes suele ser una activación emocional que se ha interpretado como un intento de restablecer la interacción con el adulto (reengage). A partir de este único indicador se ha inferido que los niños pequeños entienden las intenciones de los adultos (Melinder, Forbes, Tronick, Fikke \& Gredebäck, 2010). Sin embargo, Yazbek y D'Entremont (2006) sostienen que los niños pueden reaccionar a esta situación sin la necesidad de atribuir estados intencionales.

Tras un estudio longitudinal, estos autores demostraron que el still face effect está relacionado con la sensibilidad del infante para reconocer agentes, sin que ello implique un entendimiento de las acciones de estos agentes (Yazbek \& D'Entremont, 2006). Los niños reaccionan ante el still-face porque están experimentando una violación de expectativa en el patrón de comportamiento del agente al que están habituados, es decir, los niños esperan que los adultos se comporten como agentes sin saber, necesariamente, cuáles son las metas que dirigen sus acciones, por lo que se asombran cuando los adultos dejan de actuar como usualmente lo hacen.

Algunas investigaciones utilizan esta metodología no para inferir una comprensión intencional amplia en los niños, sino para dar cuenta de la manera como suceden las situaciones niño-cuidador, esto es, cómo los niños participan en interacciones diádicas (Lavelli \& Fogel, 2013; Provenzi, Borgatti, Menozzi \& Montirosso, 2015; Tronick et al., 2005). Inferir que el niño participa en interacciones diádicas no implica necesariamente que tenga una comprensión de las intenciones del agente, sino solo una comprensión de agentes animados 
(Tomasello et al., 2005). Esto permite afirmar que los infantes no requieren de un entendimiento de las acciones dirigidas a metas del adulto para resolver una situación de still-face effect, lo que muestra la necesidad de utilizar formas alternativas de estudiar las habilidades relacionadas con la comprensión de intenciones en edades tempranas.

\section{Metodologías Activas.}

Unsuccessful actions. La metodología de unsuccessful actions suele ser usada en edades tempranas del desarrollo (entre 6 y 18 meses) para mejorar la metodología de successful actions, procurando que la respuesta del infante se deba realmente al entendimiento de la meta. La metodología successful actions consiste en presentar una conducta completa (i.e., incluyendo el resultado de la conducta) para que sea imitada por el niño, con el objetivo de conocer si el infante entiende la meta subyacente a la conducta. Sin embargo, esta metodología no es apropiada, puesto que la imitación puede estar guiada por metas distintas a la de "completar la acción", por ejemplo, "hacer lo que hace el adulto". En la primera meta queda clara la comprensión de acciones dirigidas a metas (i.e., se entiende qué quiere hacer el adulto y se reproduce esa conducta). En la segunda, al contrario, no es clara, porque la imitación se convierte en una meta en sí misma, es decir, indudablemente el niño está cumpliendo una meta (i.e., hacer lo que hace el adulto), pero de ahí no se deduce que, necesariamente, comprenda la meta de otro individuo.

Aunque con algunas variaciones, en términos generales la metodología de unsuccessful actions consiste en que se le muestra al niño una acción (y el objeto sobre el que recae esa acción), pero no su resultado; es decir, se muestra al niño todo lo relacionado con la acción intencional, excepto la meta cumplida (Phillips \& Wellman, 2005). Ahora bien, la meta puede no conseguirse o bien porque sucede un accidente (se alcanza un resultado distinto) - lo cual corresponde a la variación denominada accidental actions - o bien porque, a pesar de que sigue intentándolo, el agente no alcanza el resultado, variación que se ha denominado trying. Independientemente de la forma en que se mida la respuesta, si el niño comprende acciones dirigidas a metas, no es necesario que observe el resultado de la conducta del agente para inferir la meta que este tiene con respecto al objeto; por tanto, se puede afirmar con mayor confianza que el niño le atribuye metas al agente.

Dado lo anterior, la metodología de unsuccessful actions ha resultado útil en el estudio de la comprensión de intenciones (Brandone, Horwitz, Aslin \& Wellman, 2014; Brandone \& Wellman, 2009; Call, Hare, Carpenter \& Tomasello, 2004; Carpenter, Akhtar \& Tomasello, 1998; Kim, Óturai. Király \& Knopf, 2015; Legerstee \& Markova, 2008; Olineck \& Poulin-Dubois, 2005; Southgate \& Csibra, 2009; Warneken \& Tomasello, 2006). Sin embargo, Schwier, van Maanen, Carpenter y Tomasello (2006) argumentan que esta metodología exige un componente imaginativo (i.e., los niños necesitan imaginar los medios adecuados para realizar la acción, así como el resultado esperado y la manera de llevarlo a cabo). Por ello, el hecho de que a los 12 meses los niños no sean capaces de llevar a cabo con éxito este tipo de tareas no significa que ellos no entiendan las metas. $\mathrm{Al}$ respecto, los autores plantean que es posible eliminar el componente imaginativo que exige la tarea experimental de unsuccessful actions, sin afectar la capacidad de una investigación para indagar sobre la comprensión que tienen los niños pequeños de las acciones intencionales. Una alternativa metodológica en respuesta a lo anterior es propuesta por los mismos autores: la metodología irrelevant actions, la cual no le exige al niño imaginar las acciones, sino elegir entre aquellas que son necesarias para cumplir la meta y aquellas que son irrelevantes.

Irrelevant actions. En esta metodología se le presenta al niño un conjunto de acciones que realiza un agente durante la consecución de una meta. Algunas de ellas son acciones irrelevantes, pues se pueden suprimir del plan de acción y aun así lograr el objetivo, y otras son acciones necesarias, sin las cuales no se puede lograr la meta. A través de esta metodología, se estudia si los niños sobreimitan (overimitation: imitan todas las acciones, incluyendo las irrelevantes) o si los niños imitan solo las acciones necesarias (imitación racional). En ese sentido, la imitación de las acciones relevantes suele interpretarse como la comprensión de la estructura intencional de la acción; en cambio, la imitación de las acciones irrelevantes se asume como la ausencia de una clara comprensión de las acciones intencionales.

Aunque este tipo de metodología se ha utilizado frecuentemente con niños entre 3 y 4,5 años (Gardiner, 2014; Kenward, 2012; Keupp, Bancken, Schillmöller, Rakoczy \& Behne, 2016; Keupp, Behne \& Rakoczy, 2013; Keupp, Behne, Zachow, Kasbohm \& Rakoczy, 2015; Király, Csibra \& Gergely 2013; Lyons, Young \& Keil, 2007; McGuigan, Whiten, Flynn \& Horner, 2007; Nielsen, Moore \& Mohamedally, 2012; Taniguchi \& Sanefuji, 2017), algunos autores introducen ciertas variaciones en las condiciones para investigar el 
desarrollo de la comprensión intencional en edades más tempranas —entre los 12 y 14 meses- (Gellén \& Buttelmann, 2017; Zmyj, Daum \& Aschersleben, 2009). Los cambios realizados repercuten, a su vez, en la interpretación de las acciones del niño. Algunas de estas variaciones consisten en situaciones en las cuales un actor exhibe acciones irrelevantes que modifican las condiciones en las que se da la posible imitación (e.g., en una demostración un actor intenta meter un perro de juguete dentro de una casa, pero la puerta está cerrada, así es que utiliza la chimenea; cuando el niño realiza la tarea, la puerta se encuentra abierta; ver Schwier et al., 2006). Se estudia, pues, la forma como un niño modifica la manera de realizar una acción cuando, a diferencia del modelo, él no se encuentra restringido. Estos datos permiten afirmar que el niño comprende la estructura intencional de la acción cuando alcanza la meta y que, además, es capaz de modificar sus acciones según las limitaciones propias y su similitud (o no) con las limitaciones de quien realiza la acción en primer lugar.

\section{Discusión}

A pesar del amplio número de estudios con metodologías pasivas que postulan un entendimiento temprano de habilidades relacionadas con la comprensión de intenciones (Biro \& Leslie, 2007; Devouche et al., 2012; Gredebäck et al., 2010; Hamlin et al., 2009; Kim \& Song, 2015; Luo, 2011; Luo \& Baillargeon, 2005; Marsh et al., 2010; Tremblay \& Rovira, 2007), no se han podido realizar afirmaciones concluyentes sobre el surgimiento de la habilidad, a causa de las limitaciones metodológicas. Ante esta dificultad, algunos estudios han intentado realizar variaciones a las metodologías activas de forma que pudieran usarse con infantes y apoyar los resultados de la habituación visual. Por ejemplo, Hamlin, Hallinan y Woodward (2008) postulan que a los siete meses ya existe un entendimiento de metas, porque los niños imitan y completan la acción de alcanzar (reaching) que ha iniciado un adulto. A pesar de que los resultados de estos autores apoyan el surgimiento temprano, Brandone y Wellman (2009) los cuestionan, porque, según ellos, la imitación de la acción de alcanzar puede deberse a una continuación del patrón comportamental iniciado por el adulto (por ser una acción conocida) y no a la comprensión de la intención propiamente dicha; es decir, el problema persiste, porque los resultados estarían dando cuenta de una reacción conductual y no de una atribución de metas. En línea con esta crítica, Southgate y Csibra (2009) establecen que, para posteriores investigaciones, es necesario presentar acciones no conocidas por los infantes, de forma que la inferencia de resultado se dé por un entendimiento de metas y no por otro tipo de asociaciones.

Lo cierto es que, por medio de metodologías diferentes, se ha llegado a conclusiones distintas sobre la misma habilidad; por ejemplo, haciendo uso de metodologías pasivas se concluye que los infantes tienen una comprensión de los agentes como dirigidos a metas alrededor de los tres a seis meses (Biro \& Leslie, 2007; Kim \& Song, 2015; Marsh et al., 2010; Tremblay \& Rovira, 2007), mientras que, a partir del uso de metodologías activas, se afirma que esta habilidad se desarrolla en edades posteriores a los 12 meses (Brandone \& Wellman, 2009; Carpenter et al., 2005; Phillips \& Wellman, 2005; Zmyj et al., 2009).

Sin embargo, indagar sobre las diferencias entre una metodología y otra difícilmente puede aportar una respuesta clara a la problemática, puesto que, como se ha mostrado, los refinamientos metodológicos no han solucionado las discusiones generadas entre autores de diferentes posturas. Concentrar todos los esfuerzos de investigación en diseñar mejores y más refinadas metodologías, y creer que de ese modo se consigue un resultado más objetivo, es una mirada miope del asunto. La metodología es un medio de aproximación a un fenómeno de estudio; más aún, ella contiene unos presupuestos conceptuales sobre el modo en que el fenómeno ha de ser concebido. En otras palabras, la metodología parece anticipar las respuestas que los investigadores esperan encontrar en los niños.

Concentrar la investigación en la aplicación arbitraria de metodologías, sin una adecuada formulación de la pregunta, limita y condiciona los resultados. Tal vez es por esto que distintas metodologías usadas para estudiar la misma habilidad dan como resultado conclusiones diferentes. Siguiendo a Vygotsky (1927/1991), "la pregunta presupone en parte la respuesta" (p. 16). Cabe, entonces, indagar qué presupuestos de base tienen los investigadores cuando sugieren el uso de una u otra metodología. Adicionalmente, debe preguntarse si la elección de la metodología a utilizar está sustentada en una comprensión amplia de la habilidad a estudiar o, en el caso contrario, si responde a un interés por "descubrir" la aparición de dicha habilidad en un momento del desarrollo del niño.

En suma, es evidente la proliferación metodológica en el campo de estudio de la comprensión de intenciones. Es posible que la tendencia de algunos autores por buscar el surgimiento de habilidades en edades cada vez más tempranas mantenga abierto un debate al respecto que no ha podido ser solventado 
mediante el refinamiento metodológico. Esto revela una necesidad de revisar no solo los aspectos metodológicos, sino también cómo se están conceptualizando las habilidades que se estudian en la comprensión de intenciones.

\section{Conclusiones}

A partir del análisis de los artículos revisados, se ha encontrado que:

1. Existen, al menos, dos tipos de metodologías en las que es posible clasificar las investigaciones actuales referentes al estudio del desarrollo temprano de la intencionalidad compartida. Por un lado, las metodologías activas, que son aquellas que utilizan el movimiento y la interacción del infante con la situación como indicadores de la habilidad estudiada. Por otro, y en un intento de responder a las primeras, surgen las metodologías pasivas. En este segundo tipo se agrupan aquellas investigaciones que utilizan indicadores observables (que disminuyen la demanda motora de la tarea) tales como reacciones visuales, emocionales o visuales y emocionales.

2. Las preferencias por una u otra metodología se deben a dos concepciones contradictorias del desarrollo. En la primera, algunos autores conciben que los niños son capaces de resolver situaciones muy complejas solo en edades tardías, considerando que no hay suficientes indicios para afirmar el desarrollo de habilidades en edades tempranas. Por tanto, su propuesta es utilizar metodologías que exigen más al niño. En la segunda, los investigadores conciben que las capacidades del niño aparecen de una vez y para siempre (i.e., no hay cambios estructurales o funcionales una vez se hace visible la habilidad), por lo que los indicios que se encuentran en edades tempranas (e.g., la discriminación visual entre dos situaciones) se interpretan como la capacidad acabada. En consecuencia, la propuesta de esta concepción consiste en utilizar metodologías que le faciliten las tareas al niño para que muestre sus verdaderas capacidades.

La discusión anteriormente descrita expone desencuentros fundamentales en la forma como se concibe el desarrollo de la intencionalidad compartida en términos metodológicos. Con ello no se está afirmando que la diversidad metodológica no sea deseable, pero sí que el criterio metodológico no basta para alcanzar una concepción teóricamente suficiente acerca del desarrollo de la intencionalidad compartida. La diversidad metodológica es fundamental allí donde ella ejerce un papel auxiliar de un planteamiento suficientemente sistemático. No se trata, pues, de anteponer la metodología al razonamiento, sino antes bien de que la metodología concrete al razonamiento en la actividad investigativa. Por ello, se glosa a Puche-Navarro (2000), "La invitación es cambiar de perspectiva, indagar sobre las condiciones que ponen en funcionamiento las capacidades del niño, antes de concluir apresuradamente que él no sabe o que no tiene la capacidad" (p. 21), aunque, a la vez, se invita a indagar lo suficiente antes de concluir de forma apresurada que el niño sabe o que ya muestra una capacidad acabada.

\section{Referencias}

Adam, M., Reitenbach, I., Papenmeier, F., Gredebäck, G., Elsner, C. \& Elsner, B. (2016). Goal saliency boosts infants' action prediction for human manual actions, but not for mechanical claws. Infant Behavior \& Development, 44, 29-37. https://doi.org/10.1016/j.infbeh.2016.05.001

Beuker, K. T., Rommelse, N. N. J., Donders, R. \& Buitelaar, J. K. (2013). Development of early communication skills in the first two years of life. Infant Behavior \& Development, 36, 71-83. https://doi.org/10.1016/j.infbeh.2012.11.001

Biro, S. \& Leslie, A. M. (2007). Infants' perception of goal-directed actions: Development through cue-based bootstrapping. Developmental Science, 10, 379-398. https://doi.org/10.1111/j.1467-7687.2006.00544.x

Brandone, A. C. (2015). Infants' social and motor experience and the emerging understanding of intentional actions. Developmental Psychology, 51, 512-523. https://doi.org/10.1037/a0038844

Brandone, A. C., Horwitz, S. R., Aslin, R. N. \& Wellman, H. M. (2014). Infants' goal anticipation during failed and successful reaching actions. Developmental Science, 17, 23-34. https://doi.org/10.1111/desc.12095

Brandone, A. C. \& Wellman, H. M. (2009). You can't always get what you want: Infants understand failed goal-directed actions. Psychological Science, 20, 85-91. https://doi.org/10.1111/j.1467-9280.2008.02246.x

Call, J., Hare, B., Carpenter, M. \& Tomasello, M. (2004). 'Unwilling' versus 'unable': Chimpanzees' understanding of human intentional action. Developmental Science, 7, 488-498. https://doi.org/10.1111/j.1467-7687.2004.00368.x

Carpenter, M., Akhtar, N. \& Tomasello, M. (1998). Fourteen- through 18-month-old infants differentially imitate intentional and accidental actions. Infant Behavior \& Development, 21, 315-330. https://doi.org/10.1016/S0163-6383(98)90009-1

Carpenter, M., Call, J. \& Tomasello, M. (2005). Twelve- and 18-month-olds copy actions in terms of goals. Developmental Science, 8, F13F20. https://doi.org/10.1111/j.1467-7687.2004.00385.x

Delafield-Butt, J. T. \& Gangopadhyay, N. (2013). Sensorimotor intentionality: The origins of intentionality in prospective agent action. Developmental Review, 33, 399-425. https://doi.org/10.1016/j.dr.2013.09.001

Devouche, E., Dominguez, S., Bobin-Bègue, A., Gratier, M. \& Apter, G. (2012). Effects of familiarity and attentiveness of partner on 6month-old infants' social engagement. Infant Behavior \& Development, 35, 737-741. https://doi.org/10.1016/j.infbeh.2012.07.021 
Feiman, R., Carey, S. \& Cushman, F. (2015). Infants representations of others' goals: Representing approach over avoidance. Cognition, 136, 204-214. https://doi.org/10.1016/j.cognition.2014.10.007

Gardiner, A. K. (2014). Beyond irrelevant actions: Understanding the role of intentionality in children's imitation of relevant actions. Journal of Experimental Child Psychology, 119, 54-72. https://doi.org/10.1016/j.jecp.2013.10.008

Gellén, K. \& Buttelmann, D. (2017). Fourteen-month-olds adapt their imitative behavior in light of a model's constraints. Child Development Research, 2017, Article ID 8080649. https://doi.org/10.1155/2017/8080649

Gredebäck, G., Fikke, L. \& Melinder, A. (2010). The development of joint visual attention: A longitudinal study of gaze following during interactions with mothers and strangers. Developmental Science, 13, 839-848. https://doi.org/10.1111/j.1467-7687.2009.00945.x

Hamlin, J. K., Hallinan, E. V. \& Woodward, A. L. (2008). Do as I do: 7-month-old infants selectively reproduce others' goals. Developmental Science, 11, 487-494. https://doi.org/10.1111/j.1467-7687.2008.00694.x

Hamlin J. K., Newman, G. E. \& Wynn, K. (2009). Eight-month-old infants infer unfulfilled goals, despite ambiguous physical evidence. Infancy, 14, 579-590. https://doi.org/10.1080/15250000903144215

Henderson, A. M. E. \& Woodward, A. L. (2011). "Let's work together": What do infants understand about collaborative goals? Cognition, 121, 12-21. https://doi.org/10.1016/j.cognition.2011.05.008

Hernik, M. \& Southgate, V. (2012). Nine-months-old infants do not need to know what the agent prefers in order to reason about its goals: On the role of preference and persistence in infants' goal-attribution. Developmental Science, 15, 714-722. https://doi.org/10.1111/j.1467-7687.2012.01151.x

Jowkar-Baniani, G., Paolozza, A., Greene, A., Cheng, C. K. \& Schmuckler, M. A. (2017). Infants' perceptions of constraints on object motion as a function of object shape. Cognition, 165, 126-136. https://doi.org/10.1016/j.cognition.2017.04.011

Kenward, B. (2012). Over-imitating preschoolers believe unnecessary actions are normative and enforce their performance by a third party. Journal of Experimental Child Psychology, 112, 195-207. https://doi.org/10.1016/j.jecp.2012.02.006

Keupp, S., Bancken, C., Schillmöller, J., Rakoczy, H. \& Behne, T. (2016). Rational over-imitation: Preschoolers consider material costs and copy causally irrelevant actions selectively. Cognition, 147, 85-92. https://doi.org/10.1016/j.cognition.2015.11.007

Keupp, S., Behne, T. \& Rakoczy, H. (2013). Why do children overimitate? Normativity is crucial. Journal of Experimental Child Psychology, 116, 392-406. https://doi.org/10.1016/j.jecp.2013.07.002

Keupp, S., Behne, T., Zachow, J., Kasbohm, A. \& Rakoczy, H. (2015). Over-imitation is not automatic: Context sensitivity in children's overimitation and action interpretation of causally irrelevant actions. Journal of Experimental Child Psychology, 130, 163-175. https://doi.org/10.1016/j.jecp.2014.10.005

Kim, E. Y. \& Song, H. -J. (2015). Six-month-olds actively predict others' goal-directed actions. Cognitive Development, 33, 1-13. https://doi.org/10.1016/j.cogdev.2014.09.003

Kim, Z., Óturai, G., Király, I. \& Knopf, M. (2015). The role of objects and effects in action imitation: Comparing the imitation of object-related actions vs. gestures in 18-month-old infants. Infant Behavior \& Development, 41, 43-51. https://doi.org/10.1016/j.infbeh.2015.07.002

Király, I., Csibra, G. \& Gergely, G. (2013). Beyond rational imitation: Learning arbitrary means actions from communicative demonstrations. Journal of Experimental Child Psychology, 116, 471-486. https://doi.org/10.1016/j.jecp.2012.12.003

Lavelli, M. \& Fogel, A. (2013). Interdyad differences in early mother-infant face-to-face communication: Real-time dynamics and developmental pathways. Developmental Psychology, 49, 2257-2271. https://doi.org/10.1037/a0032268

Legerstee, M. \& Markova, G. (2008). Variations in 10-month-old infant imitation of people and things. Infant Behavior \& Development, 31, 81-91. https://doi.org/10.1016/j.infbeh.2007.07.006

Luo, Y. (2011). Three-month-old infants attribute goals to a non-human agent. Developmental Science, 14, 453-460. https://doi.org/10.1111/j.1467-7687.2010.00995.x

Luo, Y. \& Baillargeon, R. (2005). Can a self-propelled box have a goal? Psychological reasoning in 5-month-old infants. Psychological Science, 16, 601-608. https://doi.org/10.1111/j.1467-9280.2005.01582.x

Lyons, D. E.,Young, A. G. \& Keil, F. C. (2007). The hidden structure of overimitation. Proceedings of the National Academy of Sciences of the United States of America, 104, 19751-19756. https://doi.org/10.1073/pnas.0704452104

Marsh, H. L. \& Legerstee, M. (2017). Awareness of goal-oriented behavior during infancy and early childhood, in human- and nonhuman primates. Infant Behavior \& Development, 48, 30-37. https://doi.org/10.1016/j.infbeh.2016.11.010

Marsh, H. L., Stavropoulos, J., Nienhuis, T. \& Legerstee, M. (2010). Six- and 9-month-old infants discriminate between goals despite similar action patterns. Infancy, 15, 94-106. https://doi.org/10.1111/j.1532-7078.2009.00002.x

Mastergeorge, A. M., Paschall, K., Loeb, S. R. \& Dixon, A. (2014). The still-face paradigm and bidirectionality: Associations with maternal sensitivity, self-esteem and infant emotional reactivity. Infant Behavior \& Development, 37, 387-397. https://doi.org/10.1016/j.infbeh.2014.05.006

McGuigan, N., Whiten, A., Flynn, E. \& Horner, V. (2007). Imitation of causally opaque versus causally transparent tool use by 3- and 5year-old children. Cognitive Development, 22, 353-364. https://doi.org/10.1016/j.cogdev.2007.01.001

Melinder, A., Forbes, D., Tronick, E., Fikke, L. \& Gredebäck, G. (2010). The development of the still-face effect: Mothers do matter. Infant Behavior \& Development, 33, 472-481. https://doi.org/10.1016/j.infbeh.2010.05.003

Müller, U. \& Giesbrecht, G. (2008). Methodological and epistemological issues in the interpretation of infant cognitive development. Child Development, 79, 1654-1658. https://doi.org/10.1111/j.1467-8624.2008.01216.x

Mundy, P., Block, J., Delgado, C., Pomares, Y., Van Hecke, A. V. \& Parlade, M. V. (2007). Individual differences and the development of joint attention in infancy. Child Development, 78, 938-954. https://doi.org/10.1111/j.1467-8624.2007.01042.x

Nielsen, M., Moore, C. \& Mohamedally, J. (2012). Young children overimitate in third-party contexts. Journal of Experimental Child Psychology, 112, 73-83. https://doi.org/10.1016/j.jecp.2012.01.001

Olineck, K. M. \& Poulin-Dubois, D. (2005). Infants' ability to distinguish between intentional and accidental actions and its relation to internal state language. Infancy, 8, 91-100. https://doi.org/10.1207/s15327078in0801_6

Olofson, E. L. \& Baldwin, D. (2011). Infants recognize similar goals across dissimilar actions involving object manipulation. Cognition, 118, 258-264. https://doi.org/ 10.1016/j.cognition.2010.11.012

Paulus, M. (2011). How infants relate looker and object: Evidence for a perceptual learning account of gaze following in infancy. Developmental Science, 14, 1301-1310. https://doi.org/10.1111/j.1467-7687.2011.01076.x

Perra, O. \& Gattis, M. (2012). Attention engagement in early infancy. Infant Behavior \& Development, 35, 635-644. https://doi.org/10.1016/j.infbeh.2012.06.004 
Phillips, A. T. \& Wellman, H. M. (2005). Infants' understanding of object-directed action. Cognition, 98, 137-155. https://doi.org/10.1016/j.cognition.2004.11.005

Provenzi, L., Borgatti, R., Menozzi, G. \& Montirosso, R. (2015). A dynamic system analysis of dyadic flexibility and stability across the face-to-face still-face procedure: Application of the state space grid. Infant Behavior \& Development, 38, 1-10. https://doi.org/10.1016/j.infbeh.2014.10.001

Puche-Navarro, R. (2000). Los comienzos de la experimentación y la racionalidad mejorante en el niño. En R. Puche-Navarro (Ed.), Formación de herramientas científicas en el niño pequeño (pp. 15-54). Cali, Colombia: Universidad del Valle/Arango.

Ransburg, N., Reiser, M., Munzert, J., Jovanovic, B. \& Schwarzer, G. (2017). Concurrent anticipation of two object dimensions during grasping in 10-month-old infants: A quantitative analysis. Infant Behavior \& Development, 48, 164-174. https://doi.org/10.1016/j.infbeh.2017.04.003

Schwier, C., van Maanen, C., Carpenter, M. \& Tomasello, M. (2006). Rational imitation in 12-month-old infants. Infancy, 10 , 303-311. https://doi.org/10.1207/s15327078in1003_6

Scott, R. M. \& Baillargeon, R. (2013). Do infants really expect agents to act efficiently? A critical test of the rationality principle. Psychological Science, 24, 466-474. https://doi.org/10.1177/0956797612457395

Southgate, V. \& Csibra, G. (2009). Inferring the outcome of an ongoing novel action at 13 months. Developmental Psychology, 45, 17941798. https://doi.org/10.1037/a0017197

Taniguchi, Y. \& Sanefuji, W. (2017). The boundaries of overimitation in preschool children: Effects of target and tool use on imitation of irrelevant actions. Journal of Experimental Child Psychology, 159, 83-95. https://doi.org/10.1016/j.jecp.2017.01.014

Thoermer, C., Woodward, A., Sodian, B., Perst, H. \& Kristen, S. (2013). To get the grasp: Seven-month-olds encode and selectively reproduce goal-directed grasping. Journal of Experimental Child Psychology, 116, 499-509. https://doi.org/10.1016/j.jecp.2012.12.007

Tomasello, M., Carpenter, M., Call, J., Behne, T. \& Moll, H. (2005). Understanding and sharing intentions: The origins of cultural cognition. Behavioral and Brain Sciences, 28, 675-735. https://doi.org/10.1017/S0140525X05000129

Tremblay, H. \& Rovira, K. (2007). Joint visual attention and social triangular engagement at 3 and 6 months. Infant Behavior \& Development, 30, 366-379. https://doi.org/10.1016/j.infbeh.2006.10.004

Tronick, E. Z., Messinger, D. S., Weinberg, M. K., Lester, B. M., LaGasse, L., Seifer, R. ... Liu, J. (2005). Cocaine exposure is associated with subtle compromises of infants' and mothers' social-emotional behavior and dyadic features of their interaction in the face-toface still-face paradigm. Developmental Psychology, 41, 711-722. https://doi.org/10.1037/0012-1649.41.5.711

Vygotsky, L. S. (1927/1991). El significado histórico de la crisis de la psicología: una investigación metodológica (Título original: Istoricheskii smysl psikhologicheskogo krizisa; Die Krise der Psychologie in ihrer historischen Bedeutung). En L. S. Vygotsky, Obras Escogidas, Tomo I (pp. 258-413; A. Álvarez \& P. del Río, Eds.). Madrid, España: Ministerio de Educación, Cultura y Deporte/Visor.

Warneken, F. \& Tomasello, M. (2006). Altruistic helping in human infants and young chimpanzees. Science, 311, $1301-1303$. https://doi.org/10.1126/science.1121448

Woodward, A. L. (1998). Infants selectively encode the goal object of an actor's reach. Cognition, 69, 1-34. https://doi.org/10.1016/S0010-0277(98)00058-4

Yazbek, A. \& D'Entremont, B. (2006). A longitudinal investigation of the still-face effect at 6 months and joint attention at 12 months. British Journal of Developmental Psychology, 24, 589-601. https://doi.org/10.1348/026151005X67539

Yu, C. \& Smith, L. B. (2013). Joint attention without gaze following: Human infants and their parents coordinate visual attention to objects through eye-hand coordination. PLoS ONE, 8(11), artículo e79659. https://doi.org/10.1371/journal.pone.0079659

$\mathrm{Yu}$, C. \& Smith, L. B. (2017). Hand-eye coordination predicts joint attention. Child Development, 88, $2060-2078$. https://doi.org/10.1111/cdev.12730

Zmyj, N., Daum, M. M. \& Aschersleben, G. (2009). The development of rational imitation in 9- and 12-month-old infants. Infancy, 14, 131-141. https://doi.org/10.1080/15250000802569884

Fecha de recepción: Noviembre de 2017.

Fecha de aceptación: Diciembre de 2018. 